\title{
USING HIDDEN MARKOV MODELS TO UNCOVER UNDERLYING STATES IN NEUROIMAGING DATA FOR A DESIGN IDEATION TASK
}

\author{
Goucher-Lambert, Kosa (1); McComb, Christopher (2) \\ 1: University of California, Berkeley; 2: The Pennsylvania State University
}

\begin{abstract}
Recently, design researchers have begun to use neuroimaging methods (e.g., functional magnetic resonance imaging, fMRI) to understand a variety of cognitive processes relevant to design. However, common neuroimaging analysis techniques require significant assumptions relating temporal and spatial information during model formulation. In this work, we apply hidden Markov Models (HMM) in order to uncover patterns of brain activation in a design-relevant fMRI dataset. The underlying fMRI data comes from a prior research study in which participants generated solutions for twelve open-ended design problems from the literature. HMMs are generative models that are able to automatically infer the internal state characteristics of a process by observing state emissions. In this work, we demonstrate that distinct states can be extracted from the design ideation fMRI dataset, and that designers are likely to transition between a few key states. Additionally, the likelihood of occupancy within these states is different for high and low performing designers. This work opens up the door for future research to investigate the patterns of neural activation within the discovered states.
\end{abstract}

Keywords: Design cognition, Conceptual design, Human behaviour in design, Numerical methods

\section{Contact:}

Goucher-Lambert, Kosa

University of California, Berkeley

Mechanical Engineering

United States of America

kosa@berkeley.edu

Cite this article: Goucher-Lambert, K., McComb, C. (2019) 'Using Hidden Markov Models to Uncover Underlying States in Neuroimaging Data for a Design Ideation Task', in Proceedings of the 22nd International Conference on Engineering Design (ICED19), Delft, The Netherlands, 5-8 August 2019. DOI:10.1017/dsi.2019.193 


\section{INTRODUCTION}

Understanding the cognitive processes underpinning design activity is a critical area of investigation within the design research community. Typically, these questions are answered using "black-box" experiments, in which the output of carefully designed behavioural studies are analysed in order to infer how internal processes of the mind relate to aspects of design activity. Despite their power, one limitation of design cognition studies is that the research team is left to infer the internal workings of the brain during design activity and what that may imply regarding design behaviour. In response to these limitations, an emerging research area, broadly termed "design neurocognition", seeks to apply techniques for directly measuring brain activity (e.g., with functional magnetic resonance imagingfMRI) in order to advance knowledge of the design process. Design neurocognition studies allow for researchers to more directly understand the neurocognitive processes supporting design activity. However, brain activity is itself dynamic. Classical models of analysing brain activation data rely on significant assumptions relating temporal and spatial information together in order to extract meaningful statistics linking brain activity to participant behaviour in response to experimental tasks and/or stimuli. In this work we use Hidden Markov Models (HMM), a machine learning technique, to automatically infer cognitive states in underlying fMRI data related to design cognition without prior assumptions regarding the temporal and spatial properties of the data.

The fMRI data used to explore the HMM state estimation approach in this work was collected as part of a prior study on design concept generation with and without the support of inspirational stimuli (Goucher-Lambert et al., 2019). The prior research study uncovered specific areas of brain activation correlated with productive moments of idea generation via insight, as well as a separate network correlated with continued (unsuccessful) search for concept solutions. These aforementioned areas of brain activity were determined based on regression-based estimation techniques (i.e., general linear model - GLM), in which defined model parameters were fit to the temporal brain activation data to obtain approximate brain activity levels within each voxel element. Using the HMM approach there is no assumption being made regarding the underlying model structure, and therefore we are able to automatically uncover latent patterns (states) in the design cognition fMRI data. As an initial investigation towards this broader objective, we demonstrate that distinct states can be extracted from the fMRI data and that these states provide a meaningful difference between individuals who produce high quantities and low quantities of concepts.

\section{BACKGROUND}

The methods employed in this research study builds on a variety of related works within the design research, psychology, and neuroscience literatures. This section first provides a brief introduction to efforts within the design research community utilizing fMRI, as this is the neuroimaging technique for measuring brain activation employed in this work. It is important to note that several researchers are investigating other neuroimaging modalities (e.g., Electroencephalography, functional near-infrared spectroscopy, etc.), however this is outside of the scope of the current work. Additionally, applications of HMMs both within the design community, as well as recent work applying HMMs to neuroimaging data, is introduced and discussed.

\section{1 fMRI and design cognition}

There have been a limited amount of neuroimaging studies investigating questions relevant to design. One of the first examples was research by Alexiou and colleagues who used fMRI to investigate an apartment layout task, which was generally considered to be a configuration design problem (Alexiou et al., 2009). In the work by Alexiou et al., the authors proposed a specific set of brain regions that appeared to be more active in design thinking tasks compared to problem-solving tasks; suggesting that design thinking could be distinguished from more general problem-solving. Sylcott et al. used fMRI to examine user preference judgments involving form and function trade-offs (Sylcott et al., 2013). Also, within the context of user decision-making, Goucher-Lambert et al. used fMRI to investigate product preference judgments where the environmental impact was a decision variable (Goucher-Lambert et al., 2017). The work by Goucher-Lambert et al. found areas of that areas of the brain related theory of mind reasoning were active when evaluating sustainability as a decision variable. Both the works by Sylcott $e t$ $a l$. and Goucher-Lambert et al. provide deep insights into the underlying cognitive processes involved 
when users engage in complex decision-making scenarios relevant to design. In essence, neuroimaging methods have allowed researchers to not only observe the resulting behaviour from a task/decision, but to also make inferences regarding why that decision was made.

In more recent work related to designer cognition (rather than user cognition), Goucher-Lambert and colleagues utilized fMRI to investigate design ideation and concept generation with and without the support of inspirational stimuli (e.g., text based analogies) (Goucher-Lambert et al., 2018). Goucher-Lambert et al. identified two separate patterns of brain activation associated with both the successful application of inspirational stimuli to generate design solutions via insight, as well as the open (currently unsuccessful) search for a solution (more common in the absence of inspirational stimuli). Similar to the previous work discussed in this section, the work by Goucher-Lambert et al. used regression-based contrast estimates to understand the relative differences between experimental conditions (e.g., inspirational stimuli vs. control), for both event (e.g., when idea was generated) and mixed event-block designs (e.g., average across the entire idea generation period). These analysis techniques are locked to specific time points (e.g., when ideas are generated) and therefore do not uncover connections between brain regions that may be correlated in space and in time.

\subsection{Hidden Markov models in fMRI}

As briefly mentioned previously, an HMM is a probabilistic model that is able to describe data in terms of discrete states, while also allowing for a flexible definition of state distribution. Using HMMs researchers are able to uncover recurrent patterns in brain activation data throughout entire datasets, even when the visits (or occurrences) of these patterns are relatively short in time (e.g., 5-15 seconds for fMRI data) (Vidaurre et al., 2018). Due to this flexibility, HMMs have been previously applied to fMRI data. A key assumption in the application of HMM to fMRI brain activity data is that it is reasonable to 1) represent brain activation in a discrete number of states, 2) at each point in time there is only one active state which is probabilistically assigned, and 3) that the current state being occupied is dependent on previous state occupancies (Vidaurre et al., 2017). One of the first examples of HMM applied to fMRI data was work by Anderson et al., who applied HMM to capture characteristics of mathematical problem solving (Anderson, 2012; Anderson et al., 2010, 2016). Here, the HMM was able to identify discrete states both with and without labels (defining task boundaries) that participants visited while solving mathematical problems. More recently, Baldassano and colleagues used HMM to detect event boundaries in narrative perception (e.g., blocks of a story) as shifts between patterns of brain activation that were independent of stimulus annotations (Baldassano et al., 2018). Vidaurre et al. used HMM with Human Connectome Project (HCP) data (more than 800 subjects) to show not only that brain activation data can be well represented in these cognitive states, but also that these states are hierarchically organized in time (Vidaurre et al., 2017). Here, Vidaurre et al. found that a set of meta-states emerged, for which participants were more likely to cycle within than switch between. This implied that a hierarchy of "meta-states" can represent higher or lower orders of cognitive function. Taken together, previous work in cognitive neuroscience has demonstrated HMM as a viable approach to represent brain activation data in a variety of contexts for which information regarding recurrent patterns of activity is of interest. In the work presented within this paper we are interested in uncovering both the states that emerge, as well as how people transition between different states, during design concept generation.

\subsection{Hidden Markov models in design}

The motivation for applying HMMs to neuroimaging data on design ideation comes not only from prior work on using HMMs to explore fMRI data (see Section 2.2), but also from previous work that has demonstrated HMMs as a valuable tool for capturing patterns in design process data. Prior work by the authors has applied HMMs to both assess and simulate sequential patterns of activity in the design of truss structures as well as internet-connected home cooling systems (McComb et al., 2017a, 2017b, 2018; Raina et al., 2018).

More generally, the ability of human beings to learn and employ sequences (temporal patterns of activity) has long been of interest to both psychologists (Clegg et al., 1998; Curran and Keele, 1993; Nissen and Bullemer, 1987; Perruchet and Amorim, 1992; Reed and Johnson, 1994; Willingham et al., 1989) and design researchers. Within design, the interest in sequencing spans several levels of abstraction (McComb et al., 2016). At the highest level of abstraction are design stages (e.g., customer needs assessment, conceptual design, detailed design) which also tend to occur at the longest timescales 
(Atman et al., 2007; Goldschmidt and Rodgers, 2013; Radcliffe and Lee, 1989; Stempfle and Badkeschaub, 2002). At a slightly lower degree of abstraction, and at short timescales, is the sequencing of specific design tasks, also referred to as sub-problems (Meier et al., 2007; Rogers, 1996; Sen et al., 2010; Todd, 1997; Waldron and Waldron, 1988). Sequencing at short timescales and low abstraction is a combination that is of interest because of its direct impact on the solution concepts that are developed, but this regime has been studied little in engineering design (McComb et al., 2017c). The current work, in examining the sequencing of cognitive states as imaged through fMRI, identifies and assesses a short time-scale sequence has not previously been examined in engineering design. The intersection of neuroimaging, design ideation, and analysis via hidden Markov models is a novel contribution.

\section{METHODS}

\section{1 fMRI experiment and task overview}

The underlying fMRI data utilized for this study was collected as part of a prior research study on design concept generation with and without the assistance of inspirational stimuli (Goucher-Lambert et al., 2019). The task completed in the MRI scanner was a design-thinking task, where participants developed as many solutions as possible to 12 open-ended design problems (each problem represented a separate run). For each problem, participants spent a total of 2 minutes generating solutions (Figure 1), which was separated into two 60 second blocks. The experiment was broken into three separate conditions: two where participants were given inspirational stimuli (near or far), and a third where participants were given words from the design problem (control). In the work presented in this paper, we do not separate the three experimental conditions. Three counter-balanced experimental groups provided an even distribution of problem-condition pairs for the participants. Both the problems and inspirational stimuli were taken from previous work by the authors (Goucher-Lambert and Cagan, 2019). In that work, over 1300 crowd-workers provided text-based solutions to the 12 design problems. A text-mining approach was then used to extract common/uncommon words from the crowd solutions and separate them into different distances based on word-frequency and bi-directional path length textual similarity. A full description of the design problems, inspirational stimuli, and acquisition methods for the inspirational stimuli can be found in: (Goucher-Lambert and Cagan, 2019).

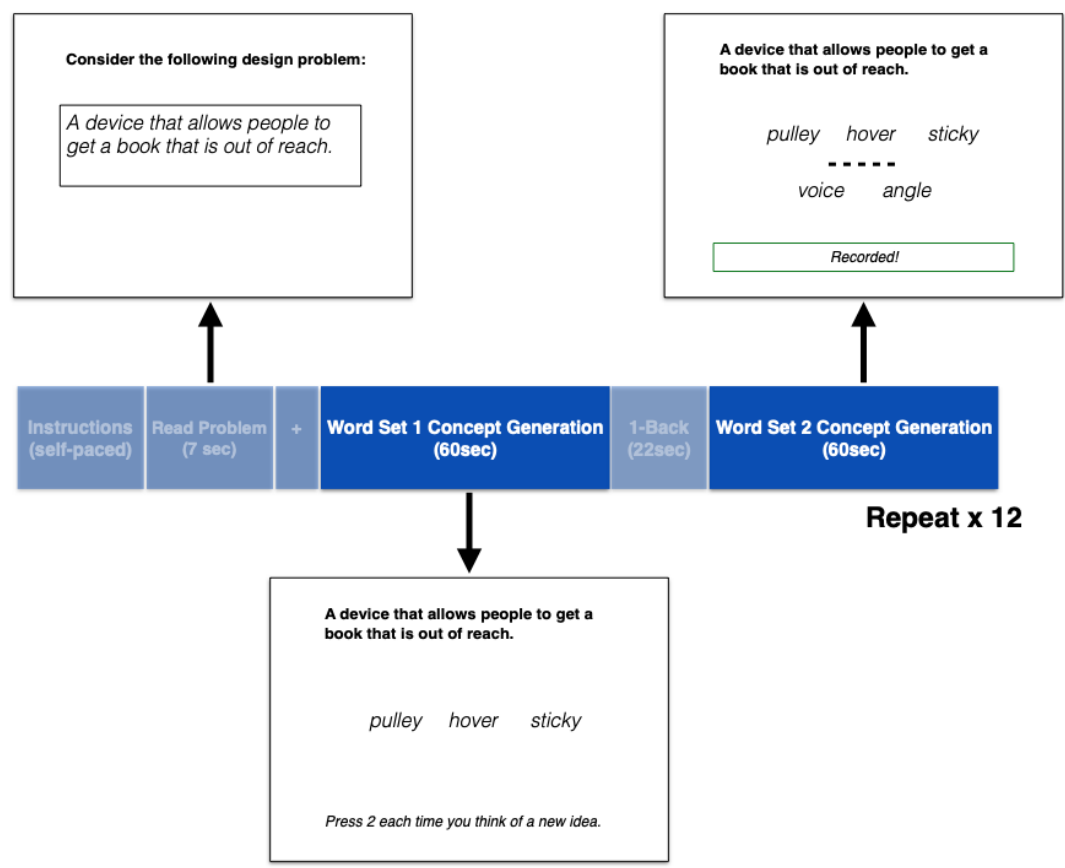

Figure 1. $f M R I$ run components and timing. Only $\mathrm{fMRl}$ images collected during concept generation periods were included in the HMM analysis.

\subsection{Collection and preprocessing of raw fMRI data}

The full details of the fMRI data acquisition parameters and processing are described in (GoucherLambert et al., 2018), and as a result will only be briefly discussed here. All 21 participants underwent a 
1-hr functional MRI brain scan where they were asked to generate solutions to 12 open-ended conceptual design problems (Section 3.1). Functional MRI data were collected from a Siemens 3T Verio MRI scanner using a 32-channel phased array head coil. Functional images were acquired using a T2*weighted multiband echo-planar imaging pulse sequence (45 oblique axial slices, in-plane resolution $3 \mathrm{~mm} \times 3 \mathrm{~mm}$, $3 \mathrm{~mm}$ slice thickness, no gap, repetition time TR=1000ms, echo time TE $=30 \mathrm{~ms}$, flipangle $=64 \mathrm{deg}$, multiband acceleration factor $=3$, matrix size $=70 \times 70$, field of view $=210 \mathrm{~mm}$, phase encoding direction $=\mathrm{P}>>\mathrm{A}$ ). 12 runs of functional data were acquired corresponding to the 12 design problems presented. Each run consisted of approximately 200 volume acquisitions, with the exact number dependent upon on self-paced portions of the run, resulting in approximately $+/-10$ volume acquisition differences between runs. T1-weighted anatomical scans were collected using the MPRAGE sequence $(0.8 \mathrm{~mm} \times 0.8 \mathrm{~mm} \times 0.8 \mathrm{~mm}, 176$ sagittal slices, $\mathrm{TR}=2300 \mathrm{~ms}$, TI $=900 \mathrm{~ms}$, flip angle $=9 \mathrm{deg}$, Generalized Autocalibrating Partial Parallel Acquisition=2). Raw fMRI data were pre-processed using the AFNI software package (March 1, 2017 version 17.0.11) (Cox, 1996). Pre-processing steps within the pipeline used for the analyses included slice scan-time correction, 3D rigid-body motion correction, high-pass temporal filtering (110s, from FSL suite (Jenkinson et al., 2012)), and spatial smoothing $(7 \mathrm{~mm}$ FWHM). An anatomical image from each subject was co-registered to his or her corresponding functional images. The structural and functional images were transformed into Talairach space with $3 \mathrm{~mm}$ isometric voxels using AFNI's auto_tlrc algorithm.

\subsection{Preparing raw fMRI data for HMM training}

The preprocessed fMRI timeseries dataset were sent through a multi-stage process to prepare them as training data for the hidden Markov model. The overall purpose of this multi stage preprocess was to reduce the entire dataset into a lower order spatial representation to more rapidly train hidden Markov models. First, every fMRI image was downsampled from the preprocessed fMRI data resolution of $54 \times 64 \times 50$ voxels $(172,800$ total voxels) to a resolution of $27 \times 32 \times 25$ (21,600 total voxels). This has been established and leveraged as in work by others as a way to avoid overfitting (Anderson, 2012). Next, principal component analysis (PCA) was applied to flattened image vectors in order to accomplish data compression, resulting in each image being represented by 50 parameters. Finally, independent component analysis (ICA) was performed using the kurtosis-maximization algorithm to produce 50 new parameters with maximal independence. A value of 50 parameters was selected based on methodology from other work (Vidaurre et al., 2017, 2018).

\subsection{Hidden Markov modelling}

A hidden Markov model was trained on the pre-processed fMRI data in order to elicit the progression of latent states. The mathematics for this modelling approach were initially established by Baum and colleagues in several seminal papers (Baum, 1972; Baum et al., 1970; Baum and Eagon, 1967; Baum and Petrie, 1966; Baum and Sell, 1968). In essence, a hidden Markov model is a generative model of a process that assumes the underlying internal state of that process is not directly observable - instead, the model attempts to infer internal state characteristics by observing emissions from those states. In this work, the states are represented by average modes of brain activation that are emitted or enacted with some degree of variance. Specifically, we trained a hidden Markov model with Gaussian emissions which is appropriate for the data used here. We used the HMM-MAR (Hidden Markov Model - Multivariate Autoregressive) toolbox ${ }^{1}$ to accomplish the analysis.

Typically, an iterative procedure is used to estimate the appropriate number of states for such a model (McComb et al., 2017a). However, because of the relatively low sample size and high number of parameters present in the data used here, there was little difference in the log-likelihood of models trained with varying numbers of hidden states. For that reason, we elected to train all hidden Markov models using 12 states, a number used in other work on neuroimaging (Vidaurre et al., 2017, 2018). The progression through states in the hidden Markov of high- and low-performing participants was also examined. This was accomplished by summing the total number of concepts generated by each participant over all runs and then assigning the participants who generated the most concepts to a highperforming group and those who developed the fewest concepts to the low-performing group.

\footnotetext{
${ }^{1}$ https://github.com/OHBA-analysis/HMM-MAR
} 


\section{RESULTS AND DISCUSSION}

Using the methods outlined in Section 3, initial analyses examined the patterns observed in both the state transitions, as well as the time-varying state assignments for the aggregated fMRI design conceptualization data. Additionally, a second analysis differentiates the study participants according to the number of ideas generated during the original cognitive study assigning each participant to either a high or low performing category. The state assignment patterns were then re-examined based on this bifurcation in order to identify states that may be associated with high (or low) rates of concept generation.

\subsection{Patterns in aggregated data}

The left side of Figure 2 shows the fraction of participants who were in a given state at any point during the study. The time variable on the x-axis concatenates Word Set 1 ( $0-60$ seconds) and Word Set 2 (60-120 seconds), such that only time periods where participants were actively attempting to generate solutions is included. Participants only rarely displayed activation in states 5, 8, 9, 10, and 12, indicating that a model with fewer states may be capable of adequately describing the data. Future work will examine whether a lower number of states or parameters may be a better fit for the neuroimaging data.

During a run, participants were most likely to be found in states $2,4,6,7$, and 11 , with State 4 having the highest likelihood of being occupied compared to any state. States 1, 6, and 11 show distinct increases in state occupation probability that occur directly after the introduction of Word Set 1 (at 0 seconds) and Word Set 2 (at 60 seconds). Recall, it is at these points in time that participants were provided with inspirational stimuli that were intended to support their idea generation productivity. States 2,4 , and 7 generally show complementing activity that occurs in the spans after Word Set introductions.

The state transition matrix for the hidden Markov model is provided on the right in Figure 2. This matrix depicts the probability that a participant will transition from one state to another between successive images. The matrix is strongly diagonal, indicating that participants are likely to stay in a single state across multiple brain image acquisitions ( $\mathrm{TR}=1$ second), rather than switch to a new state. Many of the other strong off-diagonal elements represent transitions involving with very low probabilities of occurring (e.g., states 5, 8, 9, 10, and 12), and should be interpreted as spurious quantities. One of the strongest off diagonal elements between commonly occurring states indicates a transition from state 6 to state 4 (31\% probability). Other strong off-diagonals between common states include $1 \rightarrow 6(22 \%), 2 \rightarrow 11(21 \%), 7 \rightarrow 2(16 \%)$, and $11 \rightarrow 6(17 \%)$. These connections represent an interwoven process between differing brain activation states. For example, based on the state occupancy timing, the transition from State $1 \rightarrow 6$ could be representative of early switching between encoding characteristics of the problem (State 1) to problem planning/goal setting (State 6).
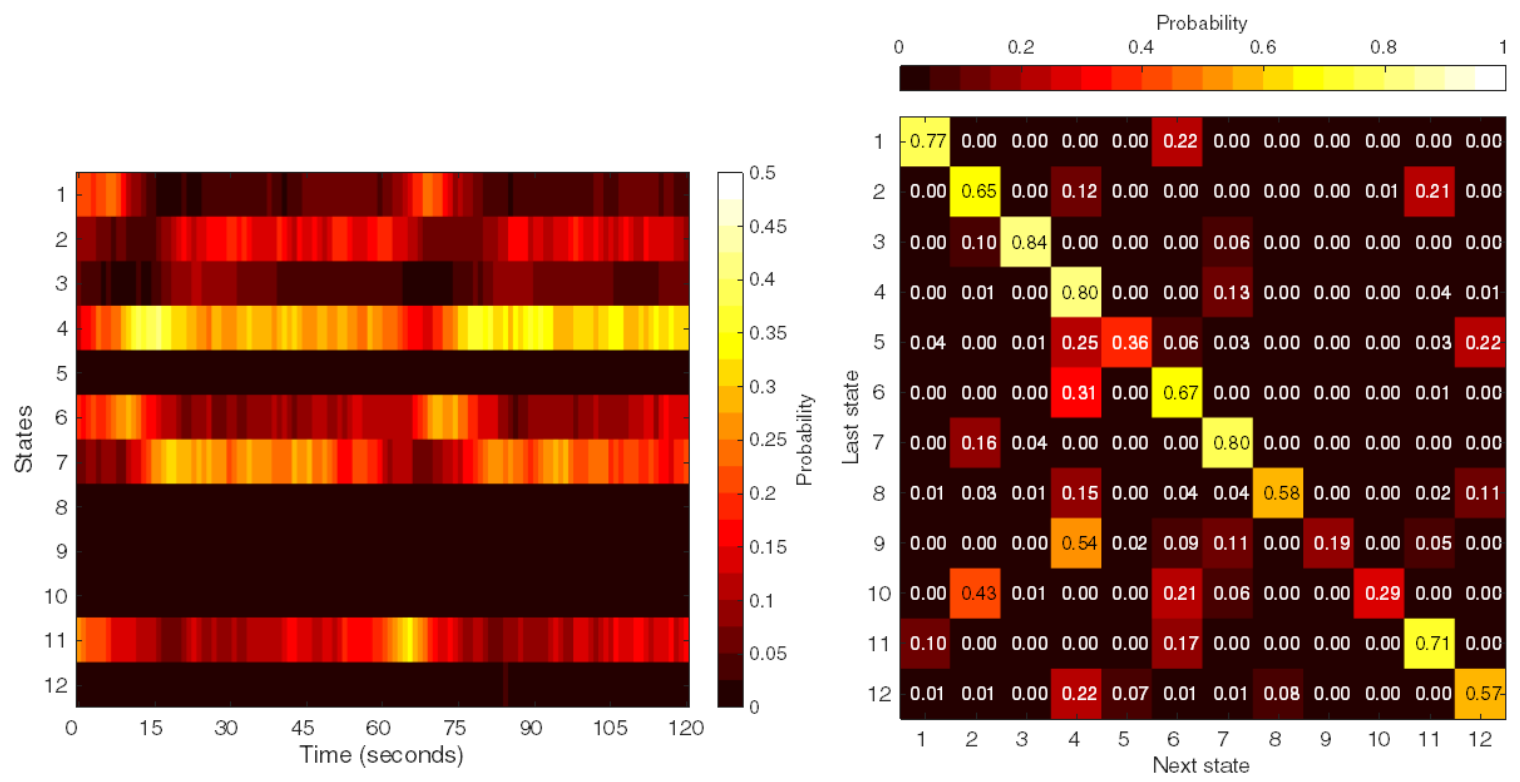

Figure 2. Results of hidden Markov model trained on aggregated data showing empirical progress through states (left) and transition matrix between states (right). 


\subsection{Performance-differentiated patterns}

Individuals were partitioned into two post-hoc conditions based on the total number of ideas they generated across all runs during the experiment. Specifically, the 10 participants who generated the most concepts across all runs (12 problems $\mathrm{x} 2$ runs per problem) were assigned to a high-performing group (average 145.5 concepts, $\mathrm{SD}=32.9$ ), and the 10 participants who generated the fewest concepts were assigned to a low-performing group (average 89.4, $\mathrm{SD}=14.7$ ). The average rate at which participants in these two groups generated ideas across an experimental run is depicted in Figure 3. On average, the high-performing individuals produce more concepts than the low-performing individual in every 15 -second block. The most pronounced differences between these two groups can be seen directly after the introduction of a new Word Set (time $=0,60$ seconds). It is also in the periods directly following the introduction of new inspirational stimuli where idea generation is most productive. Due to the differences between the high and low performing participants, one might also expect their state transitions to be different as well.

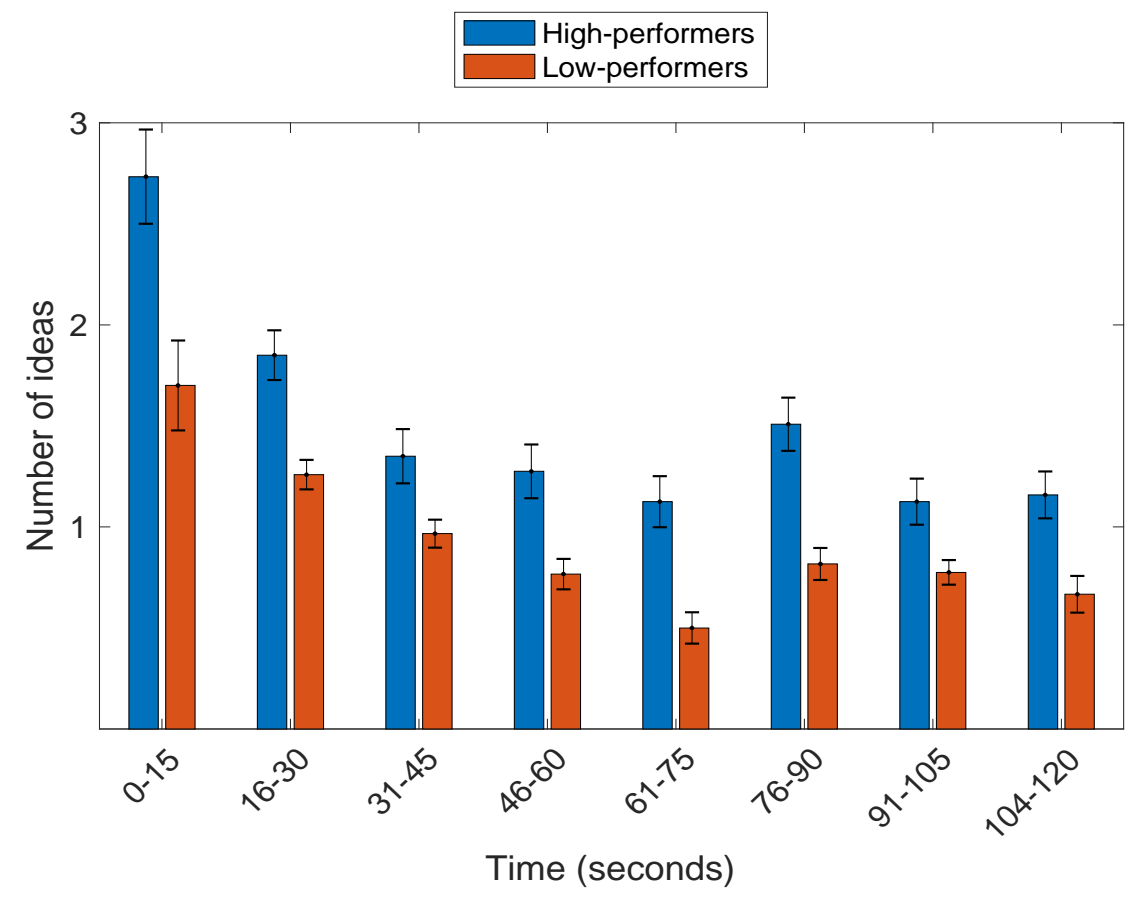

Figure 3. Average number of ideas generated per run for high-performing and lowperforming individuals (error bars indicate \pm 1 S.E.).

The empirical progress through states for each of the groups was computed with the same methodology used to produce Figure 2. The hidden Markov model was trained on the same aggregated data as presented previously, in order to ensure that the aggregated states (Figure 2) were directly comparable with the high-performing and low-performing states (Figure 4). Although some differences are apparent (particularly regarding activation in State 7), the amount of noise in these two images makes it difficult to comprehend the differences between them.

In order to better elucidate distinguishing characteristics between the high and low performing individual state occupation matrices, the difference between the two plots in Figure 4 was computed and averaged into 15-second block increments (shown in Figure 5). This highlights the major differences that are observable in a qualitative comparison of Figure 4. From this analysis it can be seen that high-performing individuals spend more time occupying State 7. Based upon this, it appears that occupying State 7 may be indicative of high performing participants and/or productive periods of design ideation more generally.

As can be seen in Figure 5, the largest difference in State 7 occupation occurs during the period between 16 seconds and 45 seconds, which is in the middle of ideation for Word Set 1 . However, there is no unique difference in ideation activity (depicted in Figure 3) that co-occurs with this difference in activation. This may indicate some degree of latency between the underlying cognitive effort that leads to increased ideation and the eventual output that results from that effort. In other words, the key to better concept generation may be a cognitive precursor to the ideas themselves. One possible 
explanation for this latency could be a delay in the hemodynamic (BOLD) response, which typically occurs 4-6 seconds after the stimuli presentation. Additionally, prior work from Goucher-Lambert et al. identified that the peak likelihood of generating a solution during Word Set 1 occurred approximately 9 seconds after the block onset (Goucher-Lambert et al., 2018). Based on this, one might expect the peak difference for State 7 to occur approximately 15 seconds into Word Set 1 , however, here we observe State 7 having a higher likelihood of occupancy for high preforming individuals 16-45 seconds after block onset. Future work is needed to identify other representative characteristics of both high and low performing participants.
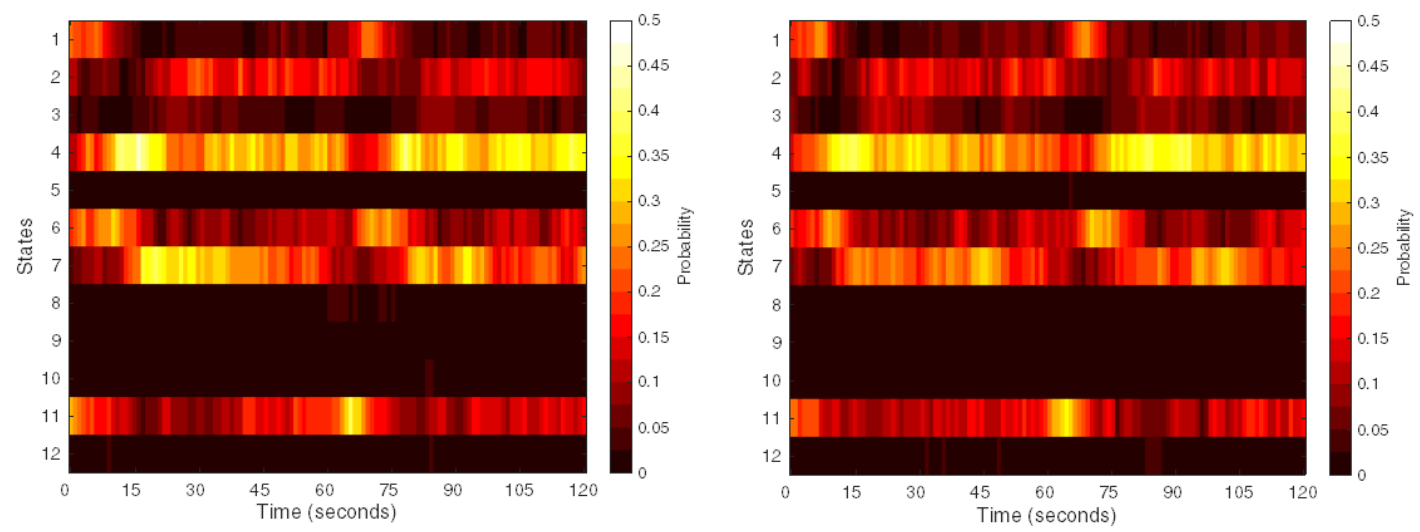

Figure 4. Empirical progress through states for high-performing individuals (left) and lowperforming individuals (right).

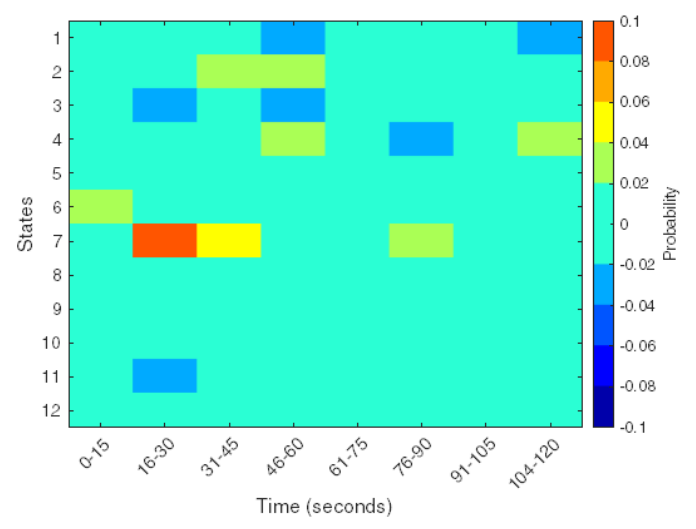

Figure 5. Differences between high- and low-performing individuals.

\section{CONCLUSION}

This work uses HMMs to automatically infer cognitive states in underlying fMRI data related to design concept generation. The underlying fMRI represents two concurrent 60 second blocks where participants were generating solutions to open ended design problems with and without the assistance of inspirational stimuli. Using this approach, the HMM model was able to represent the fMRI data in 12 distinct states, as well as demonstrate that there was switching between these states. While this work chose to represent the data using 12 states, the current findings (e.g., low occupancy likelihood in a number of states) suggest a lower number of states should be explored in future work. Additionally, partitioning the brain activation data based on high and low performing individuals (determined based upon the quantity of ideas generated) produces meaningful differences in state occupancy for one of the defined states (State 7). This implies that State 7 may be correlated with productive periods of idea generation found more often in high performing participants.

Overall, the findings presented in this work demonstrate that HMMs are well suited for identifying important characteristics representing both the spatial and temporal dynamics that occur while participants are engaged in concept generation periods. This is critical, as transitions between separate brain states are not able to be identified using classical neuroimaging analysis techniques. Ongoing and future work will investigate the patterns of neural activation that are associated with the each of the states discovered here. Additionally, future work will also consider further techniques to 
determine ICA components for the HMM input that may provide more stability (e.g., from large opensource datasets). Doing so will provide deeper insights into the cognitive underpinnings representing each of the states as well as the transitions between them relevant to design cognition during concept generation.

\section{REFERENCES}

Alexiou, K., Zamenopoulos, T., Johnson, J.H. and Gilbert, S.J. (2009), "Exploring the neurological basis of design cognition using brain imaging: some preliminary results", Design Studies, Elsevier Ltd, Vol. 30 No. 6, pp. 623-647.

Anderson, J.R. (2012), “Tracking problem solving by multivariate pattern analysis and Hidden Markov Model algorithms", Neuropsychologia, Vol. 50 No. 4, pp. 487-498.

Anderson, J.R., Betts, S., Ferris, J.L. and Fincham, J.M. (2010), "Neural imaging to track mental states while using an intelligent tutoring system", Proceedings of the National Academy of Sciences, Vol. 107 No. 15, pp. 7018-7023.

Anderson, J.R., Pyke, A.A. and Fincham, J.M. (2016), "Hidden Stages of Cognition Revealed in Patterns of Brain Activation", Psychological Science, Vol. 27 No. 9, pp. 1215-1226.

Atman, C.J., Adams, R.S., Cardella, M.E., Turns, J., Mosborg, S. and Saleem, J. (2007), “Engineering Design Processes: A Comparison of Students and Expert Practitioners", Journal of Engineering Education, Vol. 96 No. 4, pp. 359-379.

Baldassano, C., Chen, J., Zadbood, A., Pillow, J.W. and Norman, K.A. (2018), "Discovering event structure in continuous narrative perception and memory", Neuron, Vol. 95 No. 3, pp. 709-721.

Baum, L.E. (1972), “An equality and associated maximization technique in statistical estimation for probabilistic functions of Markov processes", Inequalities, Vol. 3, pp. 1-8.

Baum, L.E. and Eagon, J.A. (1967), “An inequality with applications to statistical estimation for probabilistic functions of Markov processes and to a model for ecology", Bulletin of the American Mathematical Society, Vol. 73 No. 3, pp. 360-364.

Baum, L.E. and Petrie, T. (1966), "Statistical Inference for Probabilistic Functions of Finite State Markov Chains”, The Annals of Mathematical Statistics, Vol. 37 No. 6, pp. 1554-1563.

Baum, L.E., Petrie, T., Soules, G. and Weiss, N. (1970), "A Maximization Technique Occurring in the Statistical Analysis of Probabilistic Functions of Markov Chains”, The Annals of Mathematical Statistics, Vol. 41 No. 1, pp. 164-171.

Baum, L.E. and Sell, G. (1968), "Growth transformations for functions on manifolds", Pacific Journal of Mathematics, Vol. 27 No. 2, pp. 211-227.

Clegg, B.A.B.A., Digirolamo, G.J. and Keele, S.W. (1998), "Sequence learning”, Trends in Cognitive Sciences, Vol. 2 No. 8, pp. 275-281.

Cox, R.W. (1996), "AFNI: software for analysis and visualization of functional magnetic resonance neuroimages", Computers and Biomedical Research, an International Journal, Vol. 29 No. 3, pp. 162-173.

Curran, T. and Keele, S.W. (1993), “Attentional and Nonattentional Forms of Sequence Learning”, Journal of Experimental Psychology: Learning, Memory, and Cognition.

Goldschmidt, G. and Rodgers, P.A. (2013), "The design thinking approaches of three different groups of designers based on self-reports", Design Studies, Elsevier Ltd, Vol. 34 No. 4, pp. 454-471.

Goucher-Lambert, K. and Cagan, J. (2019), "Crowdsourcing Inspiration: Using crowd generated inspirational stimuli to support designer ideation", Design Studies, http://doi.org/10.1016/j.destud.2019.01.001

Goucher-Lambert, K., Moss, J. and Cagan, J. (2017), "Inside the Mind: Using Neuroimaging to Understand Moral Product Preference Judgments Involving Sustainability (IDETC2016-59406)", ASME Journal of Mechanical Design, Vol. 139 No. 4, pp. 1-12.

Goucher-Lambert, K., Moss, J. and Cagan, J. (2018), “A neuroimaging investigation of design ideation with and without inspirational stimuli-understanding the meaning of near and far stimuli”, Design Studies, available at: http://doi.org/10.1016/j.destud.2018.07.001.

Jenkinson, M., Beckmann, C.F., Behrens, T.E.J., Woolrich, M.W. and Smith, S.M. (2012), "FSL”, NeuroImage.

McComb, C., Cagan, J. and Kotovsky, K. (2016), "Utilizing Markov Chains to Understand Operation Sequencing in Design Tasks", in Gero, J.S. (Ed.), Design Computing and Cognition '16, Springer International Publishing, Cham, Switzerland, pp. 401-418.

McComb, C., Cagan, J. and Kotovsky, K. (2017a), "Mining Process Heuristics From Designer Action Data via Hidden Markov Models”, Journal of Mechanical Design, Vol. 139 No. 11, p. 111412.

McComb, C., Cagan, J. and Kotovsky, K. (2017b), "Data on the configuration design of internet-connected home cooling systems by engineering students", Data in Brief, Vol. 14, pp. 773-776.

McComb, C., Cagan, J. and Kotovsky, K. (2017c), "Capturing Human Sequence-Learning Abilities in Configuration Design Tasks Through Markov Chains”, Journal of Mechanical Design, Vol. 139 No. 9 , p. 091101. 
McComb, C., Cagan, J. and Kotovsky, K. (2018), "Data on the design of truss structures by teams of engineering students", Data in Brief, Vol. 18, available at: http://doi.org/10.1016/j.dib.2018.02.078.

Meier, C., Yassine, A.A. and Browning, T.R. (2007), "Design Process Sequencing With Competent Genetic Algorithms", Journal of Mechanical Design, Vol. 129 No. 6, pp. 566-585.

Nissen, M.J. and Bullemer, P. (1987), “Attentional requirements of learning: Evidence from performance measures”, Cognitive Psychology, Vol. 19 No. 1, pp. 1-32.

Perruchet, P. and Amorim, M.-A. (1992), "Conscious Knowledge and Changes in Perfomance in Sequence Learning: Evidence Against Dissociation”, Journal of Experimental Psychology: Learning, Memory, and Cognition, Vol. 18 No. 4, pp. 785-800.

Radcliffe, D.F. and Lee, T.Y. (1989), “Design methods used by undergraduate engineering students”, Design Studies, Vol. 10 No. 4, pp. 199-207.

Raina, A., McComb, C. and Cagan, J. (2018), "Design Strategy Transfer in Cognitively-Inspired Agents”, ASME IDETC - Design Automation Conference, pp. 1-10.

Reed, J. and Johnson, P. (1994), “Assessing implicit learning with indirect tests: Determining what is learned about sequence structure”, Journal of Experimental Psychology: Learning, Memory, and Cognition, Vol. 20 No. 3 , pp. 585-594.

Rogers, J. (1996), “DeMAID/GA - An enhanced design manager's aid for intelligent decomposition”, 6th Symposium on Multidisciplinary Analysis and Optimization, American Institute of Aeronautics and Astronautics, available at: http://doi.org/10.2514/6.1996-4157.

Sen, C., Ameri, F. and Summers, J.D. (2010), “An Entropic Method for Sequencing Discrete Design Decisions”, Journal of Mechanical Design, Vol. 132 No. 10, p. 101004.

Stempfle, J. and Badke-schaub, P. (2002), "Thinking in design teams - an analysis of team communication", Design Studies, Vol. 23 No. 5, pp. 473-496.

Sylcott, B., Cagan, J. and Tabibnia, G. (2013), "Understanding Consumer Tradeoffs Between Form and Function Through Metaconjoint and Cognitive Neuroscience Analyses”, Journal of Mechanical Design, Vol. 135 No. 10, p. 101002.

Todd, D. (1997), Multiple Criteria Genetic Algorithms in Engineering Design and Operation, University of Newcastle.

Vidaurre, D., Abeysuriya, R., Becker, R., Quinn, A.J., Alfaro-Almagro, F., Smith, S.M. and Woolrich, M.W. (2018), "Discovering dynamic brain networks from big data in rest and task", NeuroImage, Vol. 180, pp. 646-656.

Vidaurre, D., Smith, S.M. and Woolrich, M.W. (2017), "Brain network dynamics are hierarchically organized in time”, Proceedings of the National Academy of Sciences, Vol. 114 No. 48, pp. 12827-12832.

Waldron, M.B. and Waldron, K.J. (1988), "A time sequence study of a complex mechanical system design", Design Studies, Vol. 9 No. 2, pp. 95-106.

Willingham, D.B., Nissen, M.J.J. and Bullemer, P. (1989), "On the development of procedural knowledge", Journal of Experimental Psychology: Learning, Memory, and Cognition, Vol. 15 No. 6, pp. 1047-1060.

\section{ACKNOWLEDGMENTS}

This work was supported by the Air Force Office of Scientific Research (AFOSR) under grant No. FA9550-18-1-0088. Any opinions, findings, and conclusions or recommendations expressed in this paper are those of the authors and do not necessarily reflect the views of the sponsors. 